\title{
Effects of Adding Dietary Fiber on the Gut Microbiota, Short-Chain Fatty Acids and Metabolism of Layer Chickens
}

\author{
Baosheng Sun ${ }^{1,2, \dagger}$, Linyue Hou ${ }^{1,2, \dagger}$, Yu Yang ${ }^{1, *}$ \\ ${ }^{1}$ Laboratory of Poultry Production, College of Animal Science, Shanxi Agricultural University, Jinzhong, China \\ ${ }^{2}$ Department of Modern Agriculture, Zunyi Vocational and Technical College, Zunyi, China
}

Email address:

xinxiaobao85@163.com (Baosheng Sun),sxauLynn@163.com (Linyue Hou), 345605203@qq.com (Yu Yang)

${ }^{*}$ Corresponding author

$\uparrow$ Baosheng Sun and Linyue Hou are co-first authors.

To cite this article:

Baosheng Sun, Linyue Hou, Yu Yang. Effects of Adding Dietary Fiber on the Gut Microbiota, Short-Chain Fatty Acids and Metabolism of Layer Chickens. American Journal of Life Sciences. Vol. 9, No. 5, 2021, pp. 149-156. doi: 10.11648/j.ajls.20210905.17

Received: September 30, 2021; Accepted: October 30, 2021; Published: November 5, 2021

\begin{abstract}
Dietary fiber is important for the growth performance and health of chickens. However, chickens must rely on fiber-degrading bacteria to grade fiber into monosaccharides due to a lack of endogenous fiber-degrading enzymes. Some of monosaccharides are then fermented into short-chain fatty acids (SCFAs) by SCFA-producing bacteria. SCFAs further regulate the host metabolism via special G protein-coupled receptors (GPRs) such as GPR43. In recent years, more and more research has focused on the impacts of adding dietary fiber on gut microorganisms and microbial metabolites-SCFAs of chickens. However, few works have focused on SCFA receptors and their impacts on metabolism of chickens. Understanding of this would help to explore how the dietary fiber affects chickens' metabolism via SCFAs and their receptors. Given this, three different levels $(0 \%$, $1 \%$, and $2 \%$ ) of dietary fiber-eubiotic lignocellulose were added to the feed of ISA brown hens (IBH) for 0-8 weeks, with the aim of observing the effects of adding it on the gut microbiota, SCFAs, their receptors and metabolism of chickens. The results showed that the addition of $1 \%$ significantly increased the relative abundance of SCFAs-producing bacteria Sutterella, Oscillospira and Lactobacillus panis and the production of SCFAs $(P<0.05)$. The primer sequences of the GPR 43 and reaction conditions designed in this experiment were applied to chickens. However, because the difference of the concentration of SCFAs was not great among groups, there was no significant change in the relative expression of the GPR43 mRNA in the liver and cecum $(P>0.05)$, resulting in there being no significant difference among groups in the appetite metabolism indexes including glucagon-like peptide-1 (GLP-1) and peptide tyrosine-tyrosine (PYY) and glucose metabolism indexes including blood glucose and liver glycogen of chickens $(P>0.05)$. The addition of $1 \%$ eubiotic lignocellulose is beneficial to increase the relative abundance of some SCFA-producing bacteria and the production of SCFAs at 8 weeks. The effects of added eubiotic lignocellulose on the relative expression of GPR 43 mRNA and the metabolism of chickens were slight.
\end{abstract}

Keywords: Dietary Fiber, Chicken, Eubiotic Lignocellulose, SCFA, GPR43, Gut Microbiota

\section{Introduction}

Dietary fiber is an important source for animals. However, monogastric animals such as chickens and humans must rely on gut micro to degrade fiber due to a lack of endogenous fiber-degrading enzymes [1]. Dietary fiber is broken down into monosaccharide by carbohydrate-active enzymes (CAZymes) encoded by genomes of fiber-degradation bacteria [2]. Some monosaccharides are used as energy and carbon source for poultry. A part of monosaccharides is fermented into short-chain fatty acids (SCFAs) mainly including acetate, propionate and butyrate by SCFA-producing bacteria. Bifidobacterium produces acetate using bifid-shunt [3]. Propionibacterium produces propionate via a succinate-propionate pathway [4]. Faecalibacterium and Roseburia can use acetate to generate butyrate by butyryl-CoA: acetyl-CoA transferase [5]. SCFAs can serve as an important energy source [6] and also are in control of body weight and insulin sensitivity [7]. Acetate is the main way for the host to obtain energy from dietary fiber. It can provide $1.2 \%-10 \%$ of the total energy per day for human beings. 
Propionate synthesizes glycogen in the liver. Butyrate provides energy for normal colonic epithelial cells [8] and promotes their proliferation [9]. SCFAs can increase the levels of intestinal peptides glucagon-like peptide-1 (GLP-1) and peptide tyrosine-tyrosine (PYY), thus reduce the appetite and energy intake of the host [10]. Propionate and butyrate activate intestinal gluconeogenesis (IGN) through a complementary mechanism to maintain glucose homeostasis [11]. SCFAs regulate the host metabolism, including energy metabolism, appetite regulation [12], glucose homeostasis and liver metabolism [13], which generally depend on specific G protein-coupled receptor 43 (GPR43) and GPR41 [14]. GPR41 and GPR43 have been identified in humans [7], mice [11], pigs and cattle, et al; however, the gene sequence of the SCFA receptors of chickens have not been included in the NCBI database, and related research reports are lacking. In addition, the eubiotic lignocellulose has to be proven a useful source of dietary fiber. Its proportion of total dietary fiber is as high as $85 \%$. So, no great adjustment was required in terms of the composition of the feed [15] and it was especially appropriate for experiments which were associated with the dietary fiber.

Given this, three different levels $(0 \%, 1 \%$, and $2 \%)$ of eubiotic lignocellulose as the dietary fiber source were added to the feed of chickens for $0-8$ weeks in this experiment, with the aim of observing the effects of dietary fiber on the gut microbiota and microbial metabolites-SCFAs, SCFA receptors and metabolism of chickens, and explore appropriate primer sequences of the GPR43 mRNA and PCR reaction conditions for chickens. Increasing our understanding of this would be beneficial to explore the metabolic mechansim that how dietary fiber affects the chickens' metabolism via SCFAs and their receptors and to improve the growth performance of chickens.

\section{Methods}

\subsection{Animals and Experimental Design}

This experiment was approved by the Shanxi Agricultural University Animal Experiment Ethics Committee (license number: SXAU-EAW-2017-002Chi.001). A total of 108 one-day-old ISA brown hens (IBH) with a $40 \mathrm{~g}$ average weight were chosen. Chickens were obtained commercially from Shanxi Jiabo agriculture and animal husbandry development Co., Ltd (Taigu county, Jinzhong city). Chickens were randomly divided into three groups, each group had 6 cages with 6 chickens per cage. One cage was a replicate.

Three levels of eubiotic lignocellulose OptiCell (OC) were added to the basic feed (Table 1) for 0-8 weeks. Group one (n $=36$ ) was given $1 \%$ and was called the OC-low (OL) group. Group two $(\mathrm{n}=36)$ was given $2 \%$ and was called the OC-high $(\mathrm{OH})$ group. The control group $(\mathrm{n}=36)$ was not given it, it was OC-free (OF) group. Samples were harvested to measure short-chain fatty acids and their receptors and metabolism of chickens at the end of 8 weeks. The recording of outcome measures was blinded to the treatment allocation of each experimental group.

The eubiotic lignocellulose (Beijing e-feed \& e-vet cooperation, China) was developed by Agromed Ltd. (Austria), and it is made from special fresh timber. The composition of it contains total dietary fiber (TDF) $88 \%$, crude fiber 59\%, soluble TDF $1.3 \%$, NDF 78\%, ADF $64 \%$, lignin $25 \%-30 \%$, energy $\sim 0 \%$, moisture $8 \%$, crude protein $0.9 \%$, crude ash $1.0 \%$, crude fat $0.8 \%$, minerals \& trace elements $1.3 \%$.

Table 1. Ingredients and nutrition level of feed during 0-8 weeks.

\begin{tabular}{llll}
\hline Ingredients (\%) & \multicolumn{3}{c}{ Nutrition level } \\
\hline Corn & 61.95 & ME (MJ/kg) & 12.43 \\
Soybean meal & 23.7 & Crude protein (\%) & 19.49 \\
Soybean oil & 1.1 & Crude fiber (\%) & 3.21 \\
Corn gluten meal & 4 & Crude fat (\%) & 4.27 \\
DDGS & 4 & Crude ash (\%) & 5.83 \\
Stone power & 1.8 & Ca (\%) & 1.05 \\
CaHPO 4 & 1.3 & Total P (\%) & 0.57 \\
NaCI & 0.3 & NaCI (\%) & 0.3 \\
Met & 0.2 & & \\
Lys & 0.46 & & \\
Thr & 0.09 & & \\
Multivitamin & 1 & 0.4 & \\
Minerals ${ }^{2}$ & 0.55 & & \\
Choline chloride & 0.1 & & \\
Complex enzyme & 0.05 & & \\
Total & 100 & & \\
\hline
\end{tabular}

${ }^{1}$ Feed (per kg) contains: vitamin A 2100-2500 KIU, vitamin D3 800-1240 $\mathrm{KIU}$, vitamin $\mathrm{E} \geq 5900 \mathrm{IU}$, vitamin $\mathrm{K} 3 \geq 600 \mathrm{mg}$, vitamin $\mathrm{B} 1 \geq 620 \mathrm{mg}$, vitamin $\mathrm{B} 2 \geq 1600 \mathrm{mg}$, vitamin $\mathrm{B} 6 \geq 830 \mathrm{mg}$, niacinamide $\geq 7000 \mathrm{mg}$, vitamin $B 12 \geq 4200 \mu \mathrm{g}$, pantothenic acid $\geq 2450 \mathrm{mg}$, folate $\geq 245 \mathrm{mg}$, biotin $\geq$ $35 \mathrm{mg}$.

${ }^{2}$ Feed (per kg) contains: Cu 8 mg, Fe 80 mg, Mn 60 mg, Se 0.15 mg, Zn 40 $\mathrm{mg}, \mathrm{I} 0.35 \mathrm{mg}$.

\subsection{Management}

Chickens were fed in brood cages for 0-8 weeks. Chickens were given free access to water and feed. The management of the temperature, light and humidity was conducted according to the breeding manual of IBH. No conventional immunization schedule of chickens was performed.

\subsection{Sampling}

A chicken from each replicate per group $(n=6)$ was chosen at the end of 8 weeks. The blood was collected from the wing vein after these chickens were fasted for $12 \mathrm{~h}$. The blood tube vessels were bathed in water at $37^{\circ} \mathrm{C}$ for $1 \mathrm{~h}$, followed by 3000 $\mathrm{r}$ centrifugation for $10 \mathrm{~min}$. The upper serum was absorbed into several $0.5 \mathrm{~mL}$ centrifuge tubes before being stored at $-20^{\circ} \mathrm{C}$ until further blood glucose, GLP-1 and PYY analysis. Then, they were executed with humanitarian slaughter using oral bloodletting slaughtering method. The contents of the cecum were collected into multiple cryogenic tubes and preserved at $-80^{\circ} \mathrm{C}$ until the $16 \mathrm{~S}$ rRNA gene sequence of the gut microbiota and the determination of the Concentration of SCFAs. Several pieces of liver were collected and preserved at $-20^{\circ} \mathrm{C}$ until the determination of the content of liver glycogen. As above, the cecum was collected and preserved at $-80^{\circ} \mathrm{C}$ 
until the determination of the relative expression of GPR43 mRNA.

\subsection{Determination}

\subsubsection{S rRNA Gene Sequencing}

It was performed by Genedenovo Biotechnology Ltd (Guangzhou, China) using High-Throughput Sequencing Technology. First, DNA extraction were performed using the HiPure Stool DNA Kits (Magen, Guangzhou, China). V3-V4 regions of the $16 \mathrm{~S}$ rRNA gene were amplified via PCR using primers 341F 5'-CCTACGGGNGGCWGCAG and 806R 3'-GGACTACHVGGGTATCTAAT. PCR reactions were $95^{\circ} \mathrm{C}$ for $2 \mathrm{~min}$, followed by 27 cycles at $98^{\circ} \mathrm{C}$ for $10 \mathrm{~s}, 62^{\circ} \mathrm{C}$ for $30 \mathrm{~s}, 68^{\circ} \mathrm{C}$ for $30 \mathrm{~s}$ and $68^{\circ} \mathrm{C}$ for $10 \mathrm{~min}$. Illumina Hiseq 2500 sequencing was then extracted. The datasets generated during the current study are available in the NCBI Sequence Read Archive (SRA) repository. The accession number is PRJNA732130

(http://www.ncbi.nlm.nih.gov/bioproject/732130).

Bioinformatics Analysis: (1) Quality control and reads assembly [16]. Raw tag filtering [17]. (2) Operational taxonomic units (OTUs) cluster. Effective tags were clustered into OTUs with $\geq 97 \%$ similarity [18]. (3) Bacteria biomarker features of each group were screened by LEfSe (linear discriminant analysis (LDA) effect size) software [19] and Metastats [20]. The score of LDA of certain microbes $>2$ means the difference is significant.

\subsubsection{The Concentration of SCFAs}

The concentration of SCFAs $(\mathrm{mmol} / 100 \mathrm{~g})$ in the cecum chyme was measured using the internal standard method with High Performance Gas Chromatography (HPGC) (Trace 1300, Thermo Fisher Scientific, America) [21].

\subsubsection{Relative Expression of GPR43 $\mathrm{mRNA}$}

The relative expression of GPR43 mRNA was determined using quantitative real-time PCR (qPCR).

(1) Regarding the design and synthesis of primers. At present, the gene sequences of the SCFA receptors GPR43 (FFAR2) and GPR41 (FFAR3) of chicken have not been included in the NCBI database. Only the primers of GPR43 mRNA were designed and synthesized in this experiment. The uniformity of the coding sequences of GPR43 mRNA in humans, mice, cattle and pigs included in NCBI database was $89.7 \%$ using the DNAMAN software through BLAST (Figure 1). The conserved region of the highly homologous DNA sequence was selected (red area). Primers of GPR43 were designed with Primer Premier 3.0 software. Reference gene was $\beta$-actin. The primers were synthesized by Beijing Genomics Institute (Guangdong, China) (Table 2).

uniformity $=\mathbf{8 9 . 7 3 \%}$

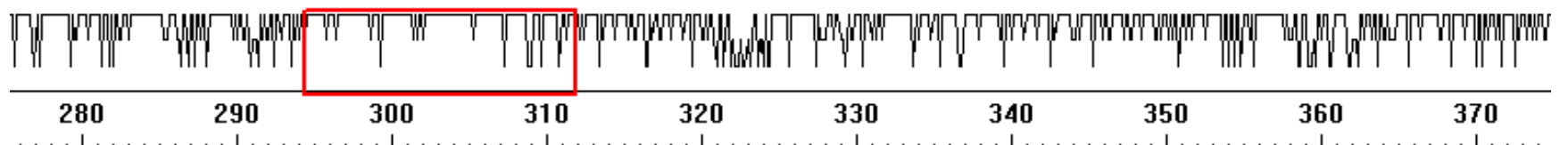

$1,1,1,1,1,1,1,1,1,1,1,1,1,1,1,1,1,1,1,1,1,1,1,1,1,1,1,1,1,1,1,1,1,1,1,1,1,1,1,1,1$

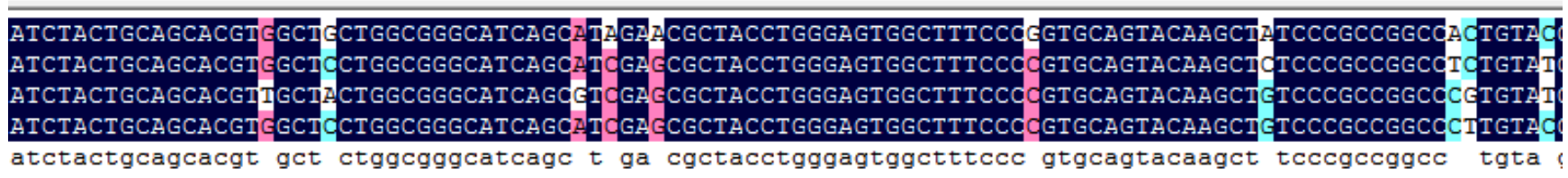

Figure 1. Blast of coding sequence of G protein-coupled receptor 43 (GPR43) in human, mouse, cow and pig.

Table 2. Primer design and synthesis.

\begin{tabular}{lll}
\hline Items & Primer & Sequence \\
\hline GRP43 & Left primer & TAGAACGCTACCTGGGAGTG \\
& Right primer & ACCAGAGCAGCGATCACTC \\
$\beta$-actin & Left primer & GAGAAATTGTGCGTGACATCA \\
& Right primer & CCTGAACCTCTCATTGCCA \\
\hline
\end{tabular}

(2) For the extraction of total RNA, liver and cecum tissues were ground to powder with liquid nitrogen, and then 1 $\mathrm{g}$ of powder sample was put into a new $1.5 \mathrm{ml}$ centrifuge tube and supplemented with $1 \mathrm{ml}$ of RNAiso Plus (Thermo Fisher Scientific, America). The total RNA was then extracted.

(3) For RNA detection, $2 \mu \mathrm{L}$ of RNA sample was taken to detect the purity and concentration by a nucleic acid protein analyzer. The range of the good-quality OD260/OD280 (R value) should be 1.8-2.2.

(4) For the first strand cDNA synthesis, the first strand of
cDNA was synthesized by reverse transcription according to the instructions of Primescript TM RT Reagent Kit with gDNA Eraser (Perfect Real Time) (Takara Bio). The steps were as follows: first, we removed genomic DNA (Table 4), with the following reaction procedure: $42^{\circ} \mathrm{C}$ for $2 \mathrm{~min}, 4^{\circ} \mathrm{C}$ for $\infty$. Second, we instigated the reverse transcription reaction (Table 3 ), with the following reaction procedure: $37^{\circ} \mathrm{C}$ for $15 \mathrm{~min}$, $85^{\circ} \mathrm{C}$ for $15 \mathrm{~s}, 4^{\circ} \mathrm{C}$ for $\infty$.

(5) For the qPCR reaction system, GPR43 mRNA was amplified with corresponding primers using the cDNA of the cecum and liver tissues as a template. The qPCR reaction system was shown in Table 4 . The $\mathrm{qPCR}$ reaction conditions were optimized, and the reaction conditions were as follows: pre-denaturation at $95^{\circ} \mathrm{C}$ for $30 \mathrm{~s}$, denaturation at $95^{\circ} \mathrm{C}$ for $5 \mathrm{~s}, 56^{\circ} \mathrm{C}$ for $30 \mathrm{~s}, 95^{\circ} \mathrm{C}$ for $15 \mathrm{~s}, 56^{\circ} \mathrm{C}$ for $1 \mathrm{~min}, 95^{\circ} \mathrm{C}$ for $30 \mathrm{~s}, 56^{\circ} \mathrm{C}$ for $15 \mathrm{~s}, 40$ cycles. 
Table 3. Reaction system.

\begin{tabular}{|c|c|c|}
\hline Items & Reagent & Dosage \\
\hline \multicolumn{3}{|c|}{ DNA remove reaction system } \\
\hline & $5 \times \mathrm{gDNA}$ Eraser Buffer & $2 \mu \mathrm{L}$ \\
\hline & gDNA Eraser & $1 \mu \mathrm{L}$ \\
\hline & Total RNA & $1 \mu \mathrm{g}$ \\
\hline & RNase Free $\mathrm{dH}_{2} \mathrm{O}$ & up to $10 \mu \mathrm{L}$ \\
\hline \multicolumn{3}{|c|}{ Reverse transcription reaction system } \\
\hline & $5 \times$ PrimeScript Buffer 2 & $4 \mu \mathrm{L}$ \\
\hline & PrimeScript RT Enzyme Mix 1 & $1 \mu \mathrm{L}$ \\
\hline & RT Prime Mix & $1 \mu \mathrm{L}$ \\
\hline & RNase Free $\mathrm{dH}_{2} \mathrm{O}$ & $4 \mu \mathrm{L}$ \\
\hline & Reaction liquid (in Table 3) & $10 \mu \mathrm{L}$ \\
\hline \multicolumn{3}{|c|}{ The qPCR reaction system } \\
\hline & $2 \times$ Es Taq Master Mix & $10 \mu \mathrm{L}$ \\
\hline & Forward Primer, $10 \mu \mathrm{mol} / \mathrm{L}$ & $1 \mu \mathrm{L}$ \\
\hline & Reverse Primer, $10 \mu \mathrm{mol} / \mathrm{L}$ & $1 \mu \mathrm{L}$ \\
\hline & cDNA template & $2 \mu \mathrm{L}$ \\
\hline & RNase Free $\mathrm{dH}_{2} \mathrm{O}$ & $6 \mu \mathrm{L}$ \\
\hline
\end{tabular}

(6) Regarding the calculation method, first, the cycle threshold (CT) of the reference gene was normalized to the $\mathrm{CT}$ value of the target gene: $\Delta \mathrm{CT}$ (treatment groups) $=\mathrm{CT}$ (experiment target gene) - CT (experimental reference gene); $\Delta \mathrm{CT}$ (control group) $=\mathrm{CT}$ (control target gene) - CT (control reference gene). The target gene was GPR43, and the reference gene was $\beta$-actin. Second, the $\Delta \mathrm{CT}$ value of the control group was normalized to the $\Delta \mathrm{CT}$ value of the experimental groups: $\Delta \Delta \mathrm{CT}=\Delta \mathrm{CT}$ (experiment groups) - $\Delta \mathrm{CT}$ (control group). Finally, the expression level ratio $2^{-\Delta \Delta}{ }^{\mathrm{CT}}$ was calculated. The value of the relative expression of GPR43 mRNA in the control group was regarded as 1, and the fold between the experiment groups and the control group was calculated.

\subsubsection{GLP-1 and PYY}

The concentration of GLP-1 and PYY in the serum was assayed by using the Chicken GLP-1 ELISA kit and PYY ELISA kit (Shanghai Huyu Biotechnology Co., Ltd. Shanghai, China), respectively. The units of them are both $\mathrm{pmol} / \mathrm{L}$.

\subsubsection{Blood Glucose and Liver Glycogen}

The concentration of blood glucose $(\mathrm{mmol} / \mathrm{L})$ in the serum was determined according to the instructions of Blood Glucose Kit (Nanjing Jiancheng Bioengineering Research Institute, Nanjing, China). The content of liver glycogen $(\mathrm{mg} / \mathrm{g})$ was determined according to the instructions of Liver Glycogen Kit (Nanjing Jiancheng Bioengineering Research Institute, Nanjing, China).

\subsection{Statistical Analysis}

Statistical analyses of indexes were performed using the ANOVA with SPSS 22.0 software. The results were expressed as the means and SEM.

\section{Results}

\subsection{Gut Microbial Composition}

The significantly dominant bacteria of the OL group were the acetate-producing genus Sutterella (0.78\%), butyrate-producing genus Oscillospira $(0.15 \%)$ and the lactate-producing species Lactobacillus panis $(0.0027 \%)$ compared with the $\mathrm{OH}(0.71 \%, 0.0068 \%, 0.00046 \%)$ and OF groups $(0.57 \%, 0.081 \%, 0.00 \%)$, respectively (Figure 2 ).

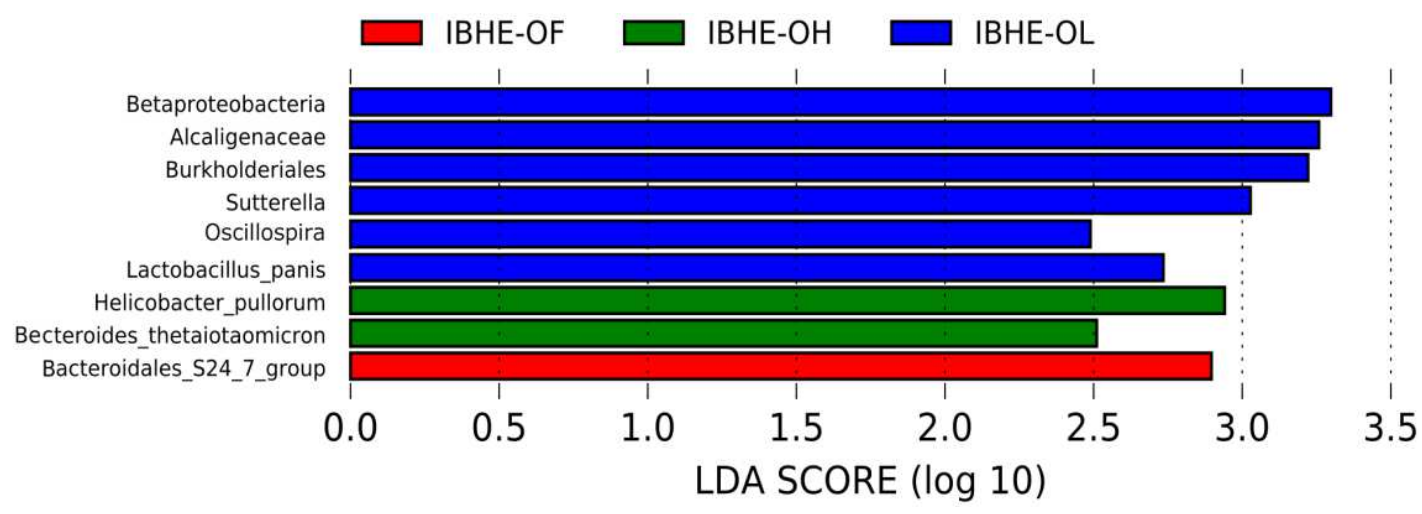

Figure 2. LDA (linear discriminant analysis) of dominant bacteria among groups.

\subsection{The Concentration of SCFAs}

SCFAs are main fermentation products of dietary fibers by gut microbiota. Therefore, the effects of the difference of the gut microbiota among groups on the concentration of SCFAs was observed. As shown in Table 4, The concentration of acetate, propionate and butyrate in the OL group was higher $(P$ $<0.01$ or 0.05$)$ than the other two groups, indicating that the addition of $1 \%$ eubiotic lignocellulose was more conducive to the production of SCFAs at 8 weeks.
Table 4. Comparison of the concentration of SCFAs among groups.

\begin{tabular}{llllll}
\hline SCFAs & IBHE-OL & IBHE-OH & IBHE-OF & SEM & $\boldsymbol{P}$-value \\
\hline Acetate & $4.07^{\mathrm{Aa}}$ & $3.01^{\mathrm{Bb}}$ & $2.42^{\mathrm{Bb}}$ & 0.25 & 0.006 \\
Propionate & $0.96^{\mathrm{Aa}}$ & $0.72^{\mathrm{ABb}}$ & $0.62^{\mathrm{Bb}}$ & 0.077 & 0.010 \\
Butyrate & $0.28^{\mathrm{a}}$ & $0.20^{\mathrm{b}}$ & $0.19^{\mathrm{b}}$ & 0.026 & 0.027 \\
\hline
\end{tabular}

Means within a line lacking a common lowercase superscript letter mean significant differences with a P-value $<0.05$, and means within a row lacking a common uppercase superscript letter mean extremely significant differences with a P-value $<0.01$. 


\subsection{Relative Expression of GPR43 mRNA}

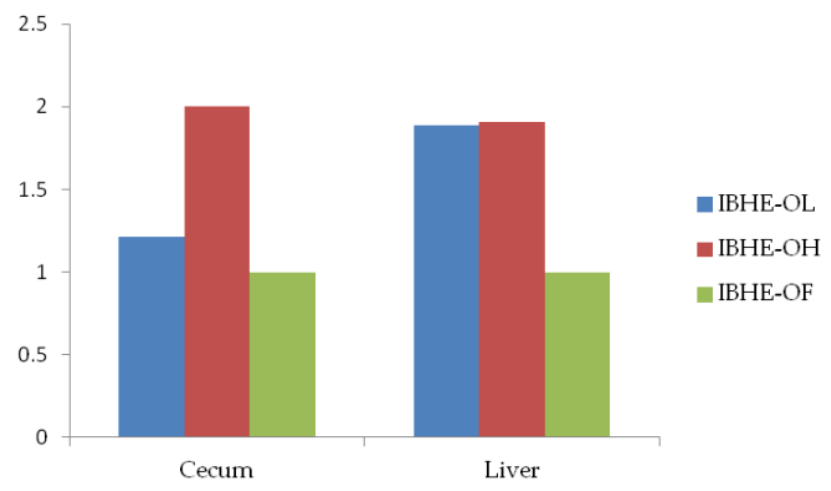

Figure 3. Histogram of the expression of $G$ protein-coupled receptor 43 (GPR43) mRNA among groups.

The SFCAs are received by the specific receptors such as GPR43 on the cell surface, thus initiating the intracellular signal transduction pathway, regulating the metabolism of the host. Therefore, the relative expression of GPR 43 mRNA of

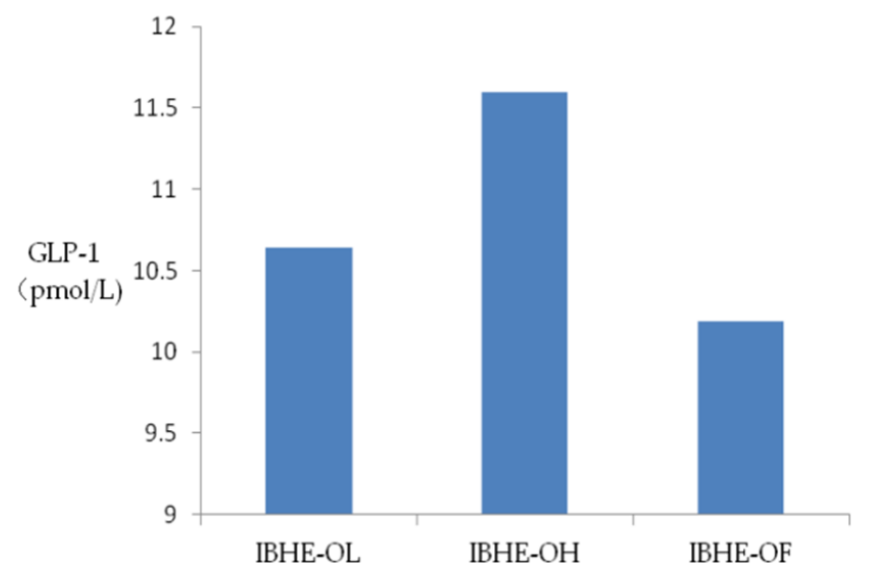

(a) Histogram of GLP-1. chickens was next determined. In this study, the range of CT values of cecum target gene GPR43 was 23-24, and that of the liver was 21-23. The CT value of cecum reference gene $\beta$-actin was $16-18$, and that of liver was $18-19$. These results showed that GPR43 mRNA was expressed well and the primer of it could be can be applied to chickens.

As shown in Figure 3, there was no difference $(P>0.05)$ among groups in the relative expression of GPR43 mRNA in the cecum and liver, but it was higher in OL and OH groups, which both had added eubiotic lignocellulose, than in the OF group.

\subsection{The Concentration of GLP-1 and PYY}

The SCFA receptors GPR41 or GPR43 can induce the release of intestinal peptides glucagon-like peptide-1 (GLP-1) and peptide tyrosine-tyrosine (PYY), making the host feel satiety and reducing appetite. Therefore, the concentration of GLP-1 and PYY were next determined.

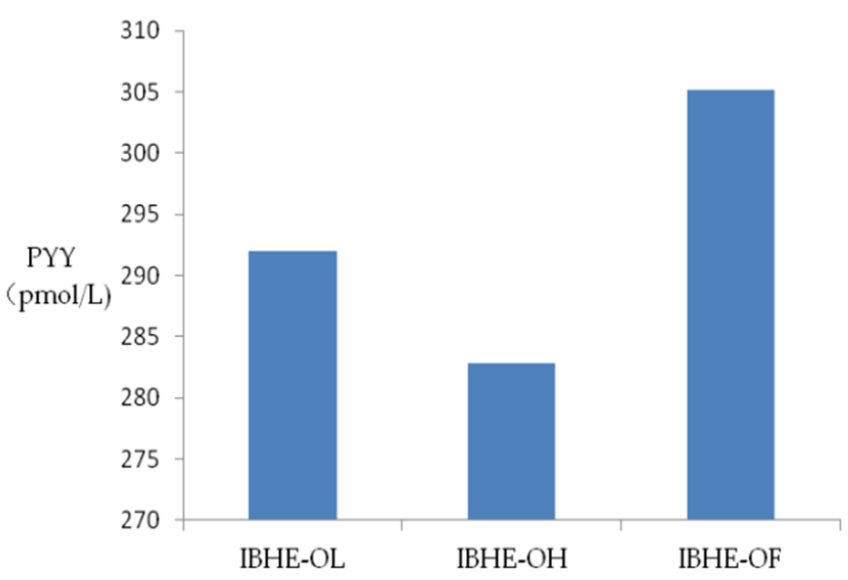

(b) Histogram of PYY.

Figure 4. Histograms of the concentration of GLP-1 and PYY among groups.

As shown in Figure 4, there was no difference $(P>0.05)$ among groups in the concentration of GLP-1 and PYY, indicating that added eubiotic lignocellulose had little effect on the satiety appetite of the host. This was consistent with there being no difference $(P>0.05)$ in the relative expression of GPR43 mRNA (Figure 3).

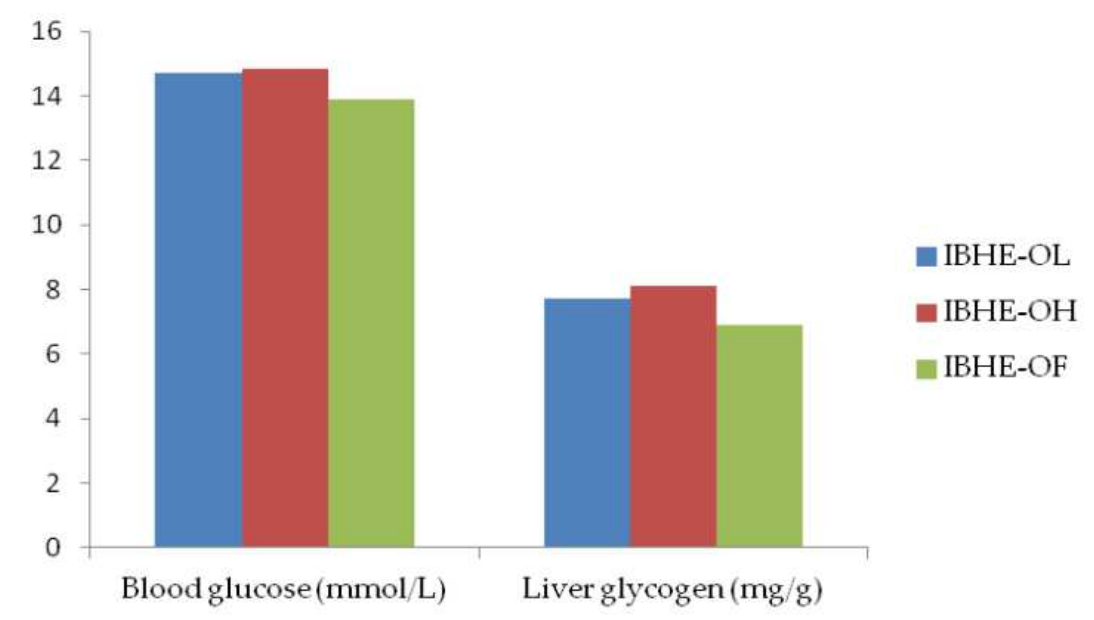

Figure 5. Histogram of content of blood glucose and liver glycogen among groups. 


\subsection{Blood Glucose and Liver Glycogen}

SCFAs also regulate the host metabolism, including glucose homeostasis and liver metabolism, which generally depend on specific SCFA receptors such as GPR43. Therefore, the blood glucose and liver glycogen of chickens were next determined.

The added eubiotic lignocellulose had no effect $(P>0.05)$ on the blood glucose and liver glycogen of chickens (Figure 5). This was consistent with there being no difference $(P>0.05)$ in the relative expression of GPR43 mRNA (Figure 4).

\section{Discussion}

\subsection{Effects of Dietary Fiber on SCFA-Producing Bacteria and SCFAs}

Dietary fiber is cleaved into monosaccharides by fiber-degradation bacteria before it is fermented into SCFAs by SCFA-producing microorganisms. Acetate, propionate and butyrate account for $90 \%-95 \%$ of SCFAs. Bifidobacterium, Sutterella and Blautia are acetate-producing bacteria. Propionate-producing bacteria include Propionibacterium, Phascolarctobacterium, Veillonella [4] and so on. Butyrate-producing bacteria include Faecalibacterium [22], Roseburia, Coprocccus [23], Oscillospira [24] and so on. The lactate produced by members of Lactobacillus can be transformed into propionate by propionate-producing bacteria [25] or butyrate by butyrate-producing bacteria $[26,5]$.

In this experiment, the relative abundances of the SCFAs-producing bacteria Sutterella, Oscillospira and Lactobacillus panis in the OL group were higher than other groups. They can effectively ferment eubiotic lignocellulose which is a synergistic combination of soluble and insoluble fiber into more lactate and SCFAs. Thus, the concentration of SCFAs in the OL group was the highest among the three groups. It suggested that the addition of $1 \%$ eubiotic lignocellulose was more conducive to increasing the abundance of certain SCFA-producing bacteria and the concentration of SCFAs at 8 weeks in this experiment.

\subsection{Effects of Dietary Fiber on GPR43, GLP-1 and PYY}

At present, the gene sequences of the SCFA receptors GPR43 (also known as FFAR2) and GPR41 (also named FFAR3) of chickens have not been included in the NCBI database. In this study, the primer sequence and reaction conditions of GPR43 gene in this experiment can be applied to chicken. The SCFAs were received by the specific receptors GPR43 or GPR41 on the cell surface [14], thus initiating the intracellular signal transduction pathway, regulating the metabolism of the host. Studies have found that SCFAs can induce the release of GLP-1 and PYY via the recognition of receptors GPR41 or GPR43, making the host feel satiety and reducing food intake [27, 28]. In this study, added eubiotic lignocellulose had no significant effect on the relative expression of GPR43 mRNA in the cecum and liver, resulting in there being no significant difference among groups in terms of the serum GLP-1 and PYY of chickens. Thus, there was no significant difference in the feed intake of chickens.

\subsection{Effects of Dietary Fiber on the Blood Glucose and Liver Glycogen}

SCFAs can also regulate the glucose homeostasis of hosts through intestinal gluconeogenesis (IGN). Gluconeogenesis is beneficial to the glucose and energy balance. Most propionate and acetate are absorbed into the portal vein [29] and enter the liver, affecting glucose metabolism. Studies have shown that the SCFAs butyrate and propionate activate IGN via complementary mechanisms [10]. Butyrate activates IGN gene expression through a cyclic adenosine monophosphate (cAMP)-dependent mechanism, and the substrate of gluconeogenesis-propionate activates IGN gene expression via a gut-brain neural circuit involving SCFA receptors [10]. In this study, there was no significant difference in blood glucose among groups, resulting in there was being no significant difference in blood glucose among groups.

Moreover, acetate and butyrate can increase the phosphorylation of AMP-activated protein kinase (AMPK) in the liver by increasing the ratio of AMP to ATP and up-regulation of peroxisome proliferator-activated receptor alpha $(P P A R \alpha)$ gene, thus increase liver glycogen storage, which is possibly mediated via a GPR41/GPR43-dependent mechanism [7]. In this study, added eubiotic lignocellulose had no significant effect on the relative expression of GPR43 mRNA, resulted in there was being no significant difference in terms of the liver glycogen among groups.

\section{Conclusions}

The addition of $1 \%$ eubiotic lignocellulose is beneficial to increase the relative abundance of some SCFAs-producing bacteria such as Sutterella and Oscillospira and the production of SCFAs at 8 weeks. The primer sequences of the GPR43 mRNA and reaction conditions designed in this experiment can be applied to chickens. The primer sequences and reaction conditions of the GPR43 mRNA for chicken are need to be further explored. The effects of added eubiotic lignocellulose on the relative expression of GPR43 mRNA were slight, resulting in the non-significant differences among groups in terms of the metabolism of chickens, including GLP-1, PYY, blood glucose and liver glycogen.

\section{Competing Interest}

All the authors do not have any possible conflicts of interest.

\section{Funding}

This research was funded by the Construction of Animal 
Husbandry Key Project of 1331 Project in Shanxi Province (J202011315) and the Construction of Double First-Class Key Disciplines of Animal Husbandry (J202111303) and the Zunyi Innovative Talent Team Training Project (Zunyi Science and Technology Talents [2021] No. 5) and the Guizhou Science and Technology Planning Project (Qian Science Contract [2020] No. 1Z001).

\section{Authors' Contributions}

All authors contributed to the study conception and design. conceptualization, YY; methodology, LH; formal analysis, BS and LH; investigation, BS and LH; resources, YY; data curation, BS and LH; writing - original draft preparation, BS and LH; writing-review and editing, BS and LH; visualization, LH; supervision, YY; project administration, YY and BS; funding acquisition, YY. All authors commented on previous versions of the manuscript and all authors read and approved the final manuscript.

\section{References}

[1] A. E. Kaoutari, F. Armougom, J. I. Gordon, D. Raoult, B. Henrissat. "The abundance and variety of carbohydrate-active enzymes in the human gut microbiota," vol. 11, Nat. Rev. Microbiol, 2013, pp. 1-9.

[2] H. J. Flint, E. A. Bayer, M. T. Rincon, R. Lamed, B. A. White, "Polysaccharide utilization by gut bacteria, potential for new insights from genomic analysis," vol. 6, Nat. Rev. Microbiol, pp. 121-131.

[3] M. Derrien, J. E. Van Hylckama Vlieg, "Fate, activity, and impact of ingested bacteria within the human gut microbiota," vol. 23, Trends Microbiol, 2015, pp. 354-366.

[4] W, Hilpert, P, Dimroth, "Conversion of the chemical energy of methylmalonyl-CoA decarboxylation into a $\mathrm{Na}^{+}$gradient," vol. 296, Nature, 1984, pp. 584-585.

[5] P, Louis, P, Young, G, Holtrop, H. J. Flint, "Diversity of human colonic butyrate-producing bacteria revealed by analysis of the butyryl-CoA: Acetate CoA-transferase gene," vol. 12, Environ. Microbiol, 2010, pp. 304-314.

[6] A. T. Reese, R. R. Dunn, "Drivers of microbiome biodiversity: a review of general rules, feces, and ignorance," vol. 9, Microbiology, 2018, pp. e01294-18.

[7] E. E. Canfora, J. W. Jocken, E. E. Blaak, "Short-chain fatty acids in control of body weight and insulin sensitivity," vol. 11, Nat. Rev. Endocrinol, 2015, pp. 577-591.

[8] D. R. Donohoe, N. Garge, X. Zhang, W. Sun, T. M. O'Connell, M. K. Bunger, et al, "The microbiome and butyrate regulate energy metabolism and autophagy in the mammalian colon," Cell Metab. vol. 13, 2011, pp. 517-526.

[9] W. E. Roediger, "Role of anaerobic bacteria in the metabolic welfare of the colonic mucosa in man," vol. 21, Gut, 1980, pp. 793-798.

[10] I. Kimura, D. Inoue, T. Maeda, T. Hara, A. Ichimura, S. Miyauchi, et al, "Short-chain fatty acids and ketones directly regulate sympathetic nervous system via $G$ protein coupled receptor 41 (GPR41)," vol. 108, PNAS, 2011, pp. 8030-8035.

[11] F. De Vadder, P. Kovatcheva-Datchary, D. Goncalves, J. Vinera, C. Zitoun, A. Duchampt, et al, "Microbiota-generated metabolites promote metabolic benefits via gut-brain neural circuits," vol. 156, Cell, 2014, pp. 84-96.

[12] G. Frost, M. L. Sleeth, M. Sahuri-Arisoylu, B. Lizarbe, S. Cerdan, L. Brody, et al, "The short-chain fatty acid acetate reduces appetite via a central homeostatic mechanism," vol. 5, Nat. Commun, 2014, pp. 1-11.

[13] T. Todesco, A. V. Rao, O. Bosello, D. J. Jenkins, "Propionate lowers blood glucose and alters lipid metabolism in healthy subjects," vol. 54, Am. J. Clin. Nutr, 1991, pp. 860-865.

[14] A. J. Brown, S. M. Goldsworthy, A. A. Barnes, M. M. Eilert, L. Tcheang, D. Daniels, et al, "The orphan G protein-coupled receptors GPR41 and GPR43 are activated by propionate and other short chain carboxylic acids," vol. 278, J. Biol. Chem, 2003, pp. 11312-11319.

[15] A. Kroismayr, S. A. Roberts. Eubiotic lignocellulose-a new tool for swine nutritionists. vol. 24, Int. Pig Top, 2010, pp. 23-25.

[16] T. Magoč, and S. L. Salzberg, "FLASH: fast length adjustment of short reads to improve genome assemblies," vol. 27, Bioinformatics, 2011, pp. 2957-2963.

[17] J. G. Caporaso, J. Kuczynski, J. Stombaugh, K. Bittinger, F. D Bushman, E. K. Costello, et al, "QIIME allows analysis of high-throughput community sequencing dat," vol. 7, Nat. Methods, 2010, pp. 335-336.

[18] R. C. Edgar, "MUSCLE, multiple sequence alignment with high accuracy and high throughput," vol. 32, Nucleic Acids Res, 2004, pp. 1792-1797.

[19] N. Segata, J. Izard, L. Waldron, D. Gevers, L. Miropolsky, W. S. Garrett, C. Hutten. "Metagenomic biomarker discovery and explanation." vol. 12, Genome Biol, 2011, pp. R60.

[20] J. R. White, N, Nagarajan, and M. Pop. "Statistical methods for detecting differentially abundant features in clinical metagenomic samples.” vol. 5, PLoS Comput. Biol, 2009, pp. e1000352.

[21] L. Hou, B. Sun, Y Yang. "Effects of added dietary fiber and rearing system on the gut microbial diversity and gut health of chicken,"vol. 10, Animals, 2010, pp. 1-22.

[22] S. H. Duncan, G. L. Hold, H. J. M. Harmsen, C. S. Stewart, H. J. Flint, "Growth requirements and fermentation products of Fusobacterium prausnitzii, and a pro-posal to reclassify it as Faecalibacterium prausnitzii gen. nov., comb. nov," vol. 52, Int. J. Syst. Evol. Microbiol, 2002, pp. 2141-2146.

[23] S. E. Pryde, S. H. Duncan, G. L. Hold, C. S. Stewart, H. J. Flint, "The microbiology of butyrate formation in the human colon," vol. 217, FEMS Microbiol. Lett, 2002, pp. 133-139.

[24] T. Konikoff, U. Gophna. "Oscillospira: a central, enigmatic component of the human gut microbiota," vol. 24, Trends Microbio, 2016, pp. 523-524.

[25] A. Koh, F. De Vadder, P. Kovatcheva-Datchary, F. Bäckhed. "From dietary fiber to host physiology: short-chain fatty acids as key bacterial metabolites," vol. 165, Cell, 2016, pp. 1332-1345. 
[26] C. V. Ferreira-Halder, V. S. F. Alessandra, S. S. Andrade, "Action and function of Faecalibacterium prausnitzii in health and disease," vol. 31, Best Practice \& Research Clinical Gastroenterology, 2017, pp. 643-648.

[27] L. Macia, J. Tan, A. T. Vieira, K. Leach, D. Stanley, S. Luong, et al, "Metabolite-sensing receptors GPR43 and GPR109A facilitate dietary fibre-induced gut homeostasis through regulation of the inflammasom," vol. 6, Nat. Commun, 2015, pp. 1-15.
[28] G. Tolhurst, "Short-chain fatty acids stimulate glucagon-like peptide-1 secretion via the G-protein-coupled receptor FFAR2," vol. 61, Diabetes, 2012, pp. 364-371.

[29] S. H. Al-Lahham, M. P. Peppelenbosch, H. Roelofsen, R. J. Vonk, K. Venema, "effects of propionic acid in humans; metabolism, potential applications and underlying mechanisms," vol. 1801, Biochim Biophys Acta, 2010, pp. 1175-1183. 